\title{
Robust Decentralized Approach to Interaction Mitigation in VSC-HVDC Grids Through Impedance Minimization
}

\author{
Adedotun J. Agbemuko ${ }^{\mathrm{a}, \mathrm{b}, *}$, José Luis Domínguez-García ${ }^{\mathrm{a}}$, Oriol Gomis-Bellmunt ${ }^{\mathrm{b}}$ \\ ${ }^{a}$ IREC Catalonia Institute for Energy Research, Jardins de les Dones de Negre 1, 2a. 08930 Sant Adrià de Besòs, Barcelona, Spain \\ ${ }^{b}$ Department of Electrical Engineering, Universitat Politècnica de Catalunya UPC, Av. Diagonal, 647, Pl. 2. 08028 Barcelona, Spain
}

\begin{abstract}
An interconnection of independently designed voltage source converters (VSCs) and their control in high voltage DC grids can result in network-level detrimental interactions and even instability. In many cases, vendors are not willing to share proprietary information about their designs in a manner that allows the entire system to be designed considering the collective dynamics. Besides, such an endeavour is not realistic. In this paper, robust network-level global controllers are proposed to decouple interacting VSCs through an impedance shaping technique. Particularly, the global controllers are designed without any need to establish the internal controller structure of any VSC which is often proprietary. Rather, the global controllers rely on the externally measurable feedback impedance of each VSC in stand-alone and the rest of the system. It is demonstrated how robust convex optimization framework can be exploited to robustly shape global feedback impedances to obtain a decoupled network. The synthesized controllers are validated through nonlinear simulations of the physical model.
\end{abstract}

Keywords: VSC-HVDC Transmission, Multivariable control, Impedance-based analysis, Robust control, H-infinity

\section{Introduction}

Over the last decade, the electric power system has witnessed a remarkable proliferation of power electronic converters in all areas of the system [1]. Particularly is the increasing adoption of voltage source converter (VSC) interfaced DC circuits and systems [2-4]. Currently, the number of VSC-based point-to-point high voltage DC (VSCHVDC) links from different manufacturers and vendors is rapidly increasing. Progressively, it is expected that these links will be interconnected to form multi-terminal DC (MTDC) grids [5-7]. On the alternative, multi-terminal grids are also expected to be developed completely from the beginning; desirably with control systems from multivendors.

An expected challenge is the issue of interoperability and coordination of independently designed and tuned converters after interconnection. An immediate consequence of these is unintended interactions between these converters at the network-level [8]. Therefore, from an external view, a network operator would have to determine how to securely operate such independently designed systems, without access to information that could be classified as proprietary. Such include the internal structure, interconnection, and architecture of control, and/or controller parameters among other information. Then, there is a requirement to determine if network-level interactions

\footnotetext{
${ }^{*}$ Corresponding author

Email address: aagbemuko@irec.cat (Adedotun J. Agbemuko)
}

warrant additional control actions. The subsequent step is the identification of the mechanism of interaction and mitigation.

Literature is extensive on network-scale interaction studies [9-13]. However, many efforts at understanding these interactions are based on the traditional state-space approach for which complete knowledge of all states (network and converters) is implicitly assumed. Moreover, state-space methods do not support independent design philosophy [14]. In reality, a manufacturer will often only provide black-box models of their devices. However, during the design phase of each converter, it is impossible to know the characteristics of all other devices that would be interconnected in the future. Hence, it is impossible to design the converter for scenarios that are uncertain.

In a multi-vendor HVDC grid, there are two broad perspectives for control: the converter itself in stand-alone (vendor), and the interconnected network of converters (operator) [15]. From the view of the vendor, the main control objectives are tracking and regulation, stand-alone stability, performance, and control channel availability for additional control actions. Alternatively, from the view of the operator, the main objectives are the stabilization of network parameters, disturbance rejection, tolerance, network characterization, and robustness. From the traditional state-space modelling and analysis, assuming all the states are known, each perspective above could be classified into local and interaction behaviours [13]. A vendor will always ensure that local objectives are met and local modes are at least stable across the expected operating 
range. However, local stability does not necessarily translate to global stability or a non-interactive network [16].

This article deals with the aforementioned objectives from the perspectives of the operator. Specifically, robust mitigation of network-level interactions between VSCs through impedance minimization. In essence, this implies a disturbance rejection problem where additional controllers decouple the network of converters (from any arbitrary interfaces) from an external view. Other methods to decouple interactions include shaping impedances by retuning controller parameters of existing local control [17]. However, this assumes that the internal structure of local control is known, and there are no complex inter-dependencies of control loops such that changing the parameters of one loop requires modifying others. Therefore, in this paper, an external view is taken where existing local controllers are assumed unchangeable and only blackbox models are available. Importantly, the local behaviour as established by the manufacturer - tracking, stand-alone stability, etc. are not adversely affected by the additional decoupling controllers. Hence, local behaviour is maintained. Besides, such a view allows the development of scalable solutions.

Impedance minimization techniques among many others fall under a large class of impedance shaping techniques. Many research efforts have exploited the modularity and scalability of impedance shaping approach from viewpoints of active damping, virtual impedance control, to methods for passivating VSCs [18-27]. More recently, impedance shaping approaches have been fitted to exploit the superiority of existing robust control methods through convex optimization [28-32]. However, except in [29] where a multi-converter system is studied, the focus of existing research is often a single converter-grid interface, and a disproportionate share of research is focused on the AC side of the VSC. In such cases, the previously described perspectives and objectives easily overlap into one with an assumption of complete knowledge of the system states. Therefore, research into network-scale impedance shaping in a multi-converter meshed DC grid is relatively unknown, even less for interaction decoupling considering independently designed local controllers. More so using only grid available measurements.

This paper presents a tractable and scalable methodology for robust decoupling of converters in VSC-HVDC grids of arbitrary structures, through impedance minimization. The method combines the input-output impedance responses of the converter and a multi-variable aggregation, and characterization of the interconnected network to determine if additional control action is required. Subsequently, interaction analysis is done to determine the mechanism of interactions, and physical objectives are transformed into frequency domain specifications. Finally, a decentralized $\mathcal{H}_{\infty}$ fixed structure convex algorithm is exploited for the robust shaping of closed-loop impedances. This paper is an extension of the initial modelling and interaction analysis efforts presented in [16, 33].

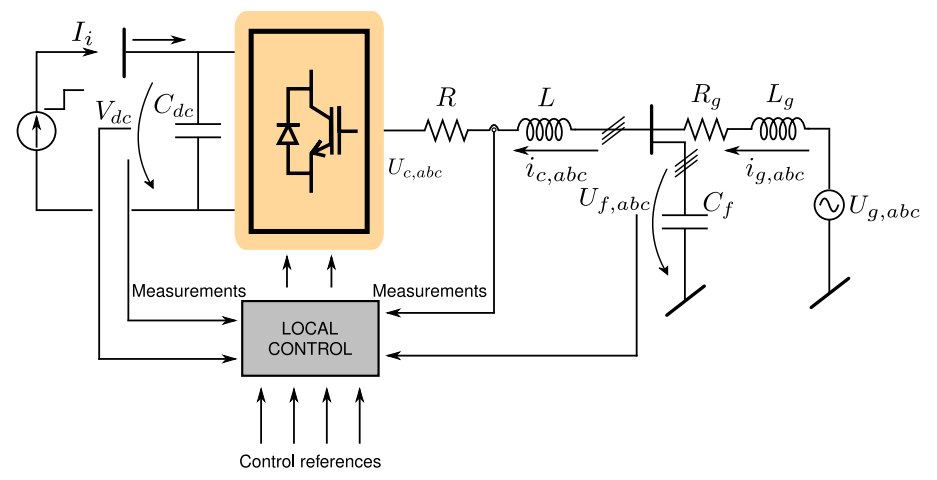

Figure 1: Schematic terminal-level diagram of a generic VSC

The main contributions of this paper are summarized below:

1. Application of input-output frequency responses of the converter and interconnected network to design coordinated decentralized network controllers.

2. Tractable formulation of the overall problem from an external view of the system with scalability to an arbitrary HVDC grid, with applicability to any system including multi-vendor systems.

3. Design of low order parametrized robust controllers with a simple structure to decouple network-scale interactions.

4. A method that incorporates flexibility for different approaches from a multi-variable point of view.

This paper is organized as follows. Section 2 presents the numerically estimated input-output impedance of a controlled VSC assuming black-box models and verified with analytical derivations. Section 3 presents the network aggregation and reformulation of the system into two partitions for control design and the generalized procedures. Then, Sections 4 and 5 applies the procedure to design global controllers for a meshed HVDC grid.

Table 1: System Parameters

\begin{tabular}{cccc}
\hline \hline Parameter & Value & Parameter & Value \\
\hline Rated power & $800 \mathrm{MVA}$ & $L$ & $42 \mathrm{mH}$ \\
DC voltage & $400 \mathrm{kV}$ & $C_{f}$ & $10 \mu \mathrm{F}$ \\
AC voltage & $220 \mathrm{kV}(\mathrm{p}-\mathrm{p})$ & $R_{g}$ & $0.048 \Omega$ \\
$C_{d c}$ & $150 \mu \mathrm{F}$ & $L_{g}$ & $15 \mathrm{mH}$ \\
$R$ & $0.242 \Omega$ & & \\
\hline
\end{tabular}

\section{Simplified Input-output Impedance Response of a Controlled VSC}

From a black-box overview, the terminal-level block diagram of a synchronous reference frame VSC [34], is shown 


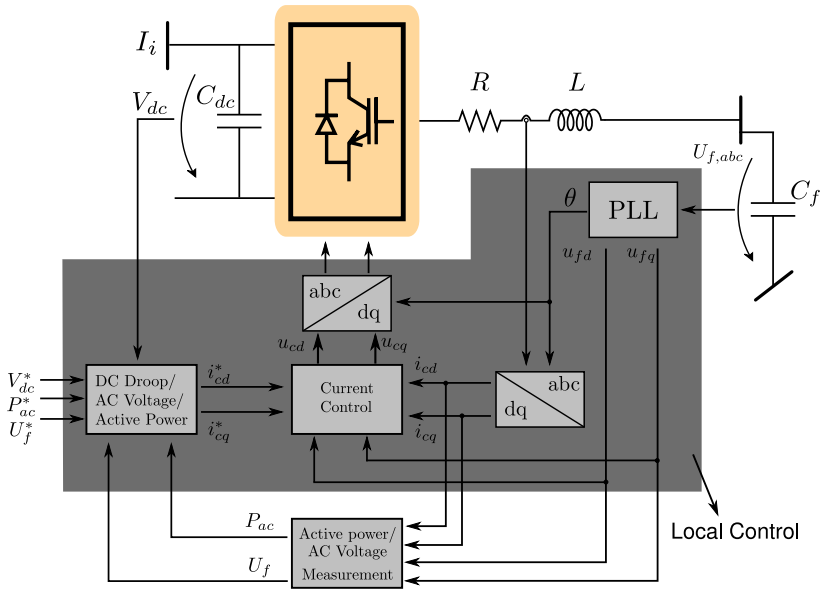

Figure 2: Local control structure of a VSC-station

in Fig. 1. The system parameters of the VSC as shown in Fig. 1 is provided in Table 1 . The aim here is to obtain the input-output feedback impedance transfer function of the converter on the DC side in the form

$$
Z_{o c, i}(s)=\frac{\Delta V_{d c, i}}{\Delta I_{i}}=\frac{b_{m} s^{m}+\cdots+b_{1} s+b_{0}}{a_{n} s^{n}+a_{n-1} s^{n-1}+\cdots+a_{1} s+a_{0}}
$$

where $Z_{o c, i}$ is the feedback impedance and equivalent input-output response of the $\mathrm{VSC}^{1}, \Delta V_{d c, i}$ is the DC-bus voltage response to local disturbance (without interconnection), $\Delta I_{i}$ is the bus current changes during disturbance, and the coefficients of $Z_{o c, i}$ may be arbitrary. In this paper, a numerically estimated transfer function from input-output time-domain simulation responses given the pre-defined local control strategy is compared to an analytical derivation. To facilitate the analytical derivations, Fig. 2 shows the control block layout and internal interconnections of the generic VSC. However, these interconnections are only for the analytical derivations and a knowledge of this structure from a network perspective is not required. The desired time responses of each control system as shown is provided in Table 2 and can be used in computing the controller parameters. All other passive subsystems and components such as lines and cables can be modelled in the same manner with transfer functions. Frequency-dependent transfer function models for cables are widely available from literature [35].

\subsection{Impedance Response of a Direct-Voltage Controlled VSC}

For a VSC in direct voltage local control mode, a simplified analytical derivation can be obtained given two simplifying assumptions:

1. The connected AC grid is fairly strong, with SCR $>$ 3. Then, it may be assumed that the point

\footnotetext{
${ }^{1}$ In practice, $Z_{o c, i}$ is a set of transfer functions rather than one function.
}

Table 2: Control Parameters

\begin{tabular}{ccc}
\hline Controller & Time constant & Damping ratio \\
\hline AC-side Current & $1.5 \mathrm{~ms}$ & 0.7071 \\
DC-side voltage & $15 \mathrm{~ms}$ & 0.7071 \\
AC-side voltage & $100 \mathrm{~ms}$ & 0.7071 \\
Active power & $20 \mathrm{~ms}$ & 0.7071 \\
Phase-locked loop & $20 \mathrm{~ms}$ & 0.7071 \\
\hline
\end{tabular}

of common coupling (PCC) bus-voltage magnitude $U_{f}=\sqrt{u_{f d}^{2}+u_{f q}^{2}}$ is constant at the defined value such that,

$$
\begin{aligned}
u_{f q} & \approx 0 \\
\Longrightarrow U_{f} & \approx u_{f d}, \Longrightarrow \Delta u_{f d} \approx 0 .
\end{aligned}
$$

where $u_{f d}$ and $u_{f q}$ are the PCC-bus $d$ and $q$ axes alternating voltages. That is, the impact of alternating voltage controller (AVC) vanishes and the $\mathrm{AC}$ side is fairly decoupled from the DC side (more details can be found in [16]).

2. If the above holds, the reactive current reference can be assumed constant.

It is important to note that the above assumptions only allow to neglect the impact of $\mathrm{AC}$ dynamics on the $\mathrm{DC}$ side. However, this does not hold if the connected $\mathrm{AC}$ grid at the PCC is weak; in which case the interaction functions between $\mathrm{AC}$ and $\mathrm{DC}$ variables must be considered in detail. Following this approach, the resultant simplified direct voltage control loop is shown in Fig. 3. The closed-loop expression of the $i^{\text {th }}$ direct voltage-controlled VSC can be derived from the block diagram as

$$
\begin{aligned}
& V_{d c, i}=H_{c l, i}^{v}(s) V_{d c, i}^{*}+Z_{o c, i}^{v}(s) I_{i} \\
& \Longrightarrow \Delta V_{d c, i}=Z_{o c, i}^{v}(s) \Delta I_{i}
\end{aligned}
$$

where $H_{c l, i}^{v}(s)$ is the closed-loop reference-to-output transfer function, $V_{d c, i}^{*}$ is the voltage reference obtained from power flow, $I_{i}$ is the total DC-bus current flowing into the converter, $K_{v}(s)$ the direct voltage controller (DVC), and $Z_{o c, i}^{v}(s)$ is the input-output impedance of a direct voltage controlled converter in the same form as (1). Analytically it can be derived as

$$
Z_{o c, i}^{v}(s)=\frac{Z_{p i}(s)}{1+H_{o l, i}^{v}(s)}
$$

with

$$
H_{o l, i}^{v}(s)=c K_{v}(s) h_{c l}^{i} Z_{p i}(s) ; \quad c=\frac{u_{f d, i}^{0}}{k V_{d c, i}^{0}}
$$

where $h_{c l}^{i}$ is the closed-loop reference-to-output transfer function of the inner-loop, $u_{f d, i}^{0}$ and $V_{d c, i}^{0}$ are the operating points of the PCC and DC-bus voltages respectively, and 


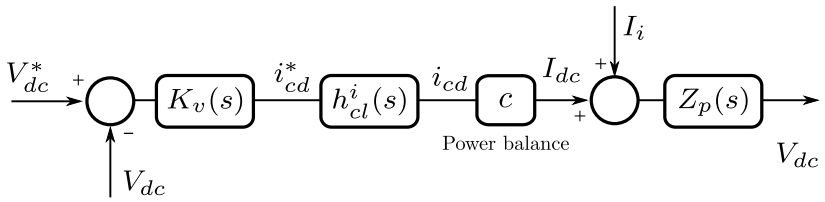

Figure 3: Simplified closed-loop block diagram of the DVC loop

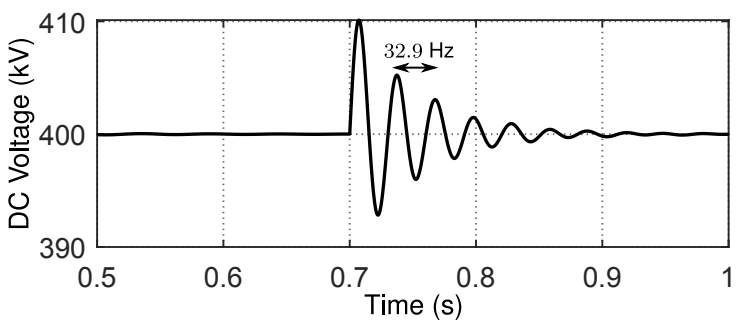

Figure 4: Direct voltage response to step input in bus power for a direct voltage controlled VSC

$Z_{p i}(s)$ is the equivalent physical impedance on the DC side of the VSC. It is important to reiterate that the main interest is the final form of the input-output impedance $Z_{o c, i}(s)$ and not the structure of internal control blocks or components that make it up. Hence, it is assumed that the inner-loop for instance is properly tuned and fixed-it's response cannot be changed, and the entire local control including the DVC is stable.

The circuit schematic for obtaining input-output timedomain responses is similar to Fig. 1. For a $150 \mathrm{MW}$ step input in inversion at $0.7 \mathrm{~s}$, Fig. 4 shows the timedomain response of the terminal direct voltage to the step change. Clearly, there is one stable local oscillatory mode around $32.9 \mathrm{~Hz}$ from which a transfer function can be estimated. In this work, the spectral analysis of the inputoutput time-domain response is applied in estimating the frequency response; other methods are available from literature. A comparison between the analytically derived input-output impedance response and the numerically estimated response from the shown time-domain simulation is shown in Fig. 5. It can be seen that there is a clear match between the numerically estimated response from the detailed model and the analytically derived response given the assumptions made. Therefore, this is the nominal feedback impedance equivalent of a direct voltage controlled VSC as seen from the external terminals.

From the analytical derivations, it can be seen that the input-output impedance depends on the operating point. To establish the impacts, Fig. 6 shows the responses of the analytically derived function across the expected operating range of the VSC from -800 MW - $800 \mathrm{MW}$. Importantly, it shows that the local oscillatory frequency remains the same, only the magnitude differs across the operating range; differences in phase is limited to the region around the local mode. However, models adopted at the networklevel will depend on the exact power flow, but the eventual transfer function will be within the shown set.

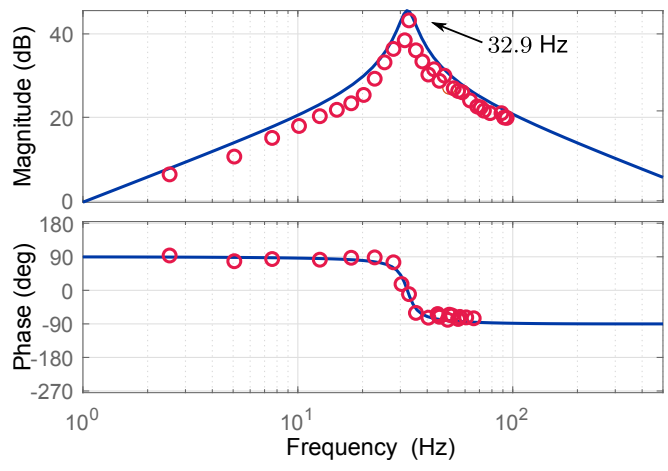

Figure 5: Nominal frequency response of the input-output impedance of a direct voltage controlled VSC: analytical (solid blue), numerical estimation (circled red)

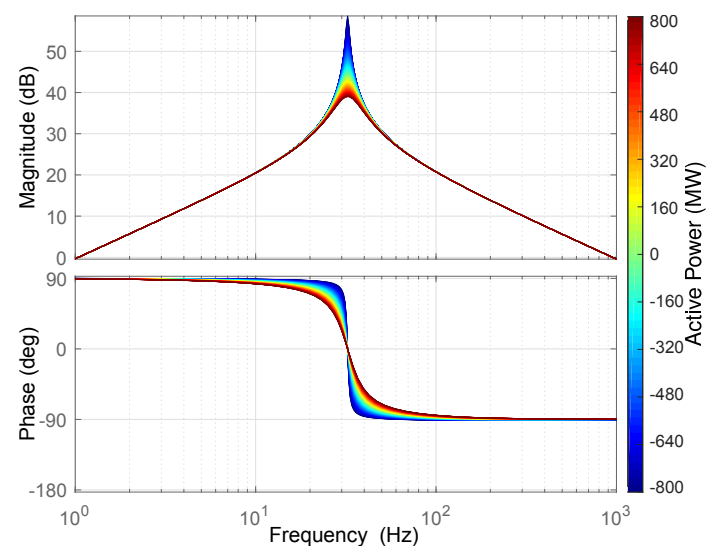

Figure 6: Sensitivity of impedance response to varying operating points

\subsection{Impedance Response of a Droop/Active Power Con- trolled VSC}

As an alternative to direct voltage control, a VSC may be equipped with droop/active power control. Both modes can be analytically combined, with the droop gain determining whether the controller is in active power or droop mode $-R_{d c, i}=0$ implies active power, and $R_{d c, i} \gg 0$ implies droop control. From the perspective of a manufacturer, [36] discusses how to design droop gains from an impedance viewpoint to meet minimum performance set by an operator. With similar assumptions as previously, Fig. 7 shows the simplified block diagram of a droop/active power controlled VSC. In addition, if the VSC is assumed lossless,

$$
P_{a c}=P_{d c}
$$

where $P_{d c}$ is the DC-bus power and $P_{a c}$ is the AC-bus power. Due to the presence of the nonlinear power variable, linearization around an operating point must be done. Thus, at the $j^{\text {th }}$ VSC

$$
\Delta P_{a c, j}=\Delta P_{d c, j}=V_{d c, j}^{0} \Delta I_{d c, j}+I_{d c, j}^{0} \Delta V_{d c, j}
$$

where $I_{d c, j}$ is the converter injection current balanced with the AC side. Following the block diagram, the closed-loop 


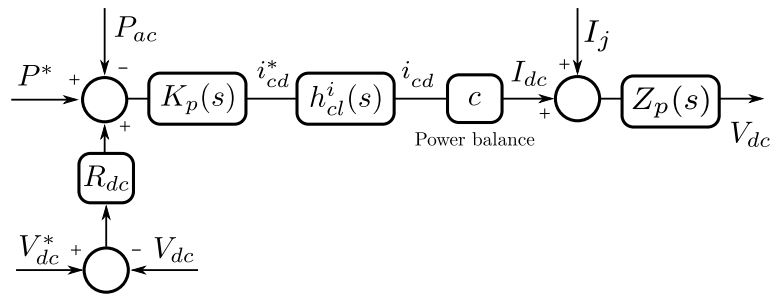

Figure 7: Simplified closed-loop block diagram of the droop/active power loop

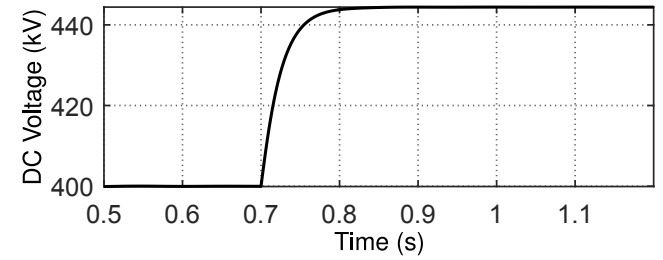

Figure 8: Direct voltage response to step input in bus power for a droop/active power controlled VSC

response can be derived in a similar form to the direct voltage VSC as

$$
\Delta V_{d c, j}=\underbrace{\frac{Z_{p j}(s)}{1+Z_{p j}(s)\left(\frac{c K_{p}(s) h_{c l}^{i}(s)\left(I_{c c, j}^{0}+R_{d c, j}\right)}{1+c K_{p}(s) h_{c l}^{i}(s) V_{d c, j}^{d}}\right)}}_{Z_{o c, j}^{d_{p}}(s)} \Delta I_{j}
$$

where $R_{d c, j}$ is the droop gain, $K_{p}(s)$ is the active power compensator, and $Z_{o c, j}^{d p}(s)$ is the imposed DC impedance by a droop/active power controlled VSC.

In a similar manner as the direct voltage VSC, Fig. 8 shows the time-domain response to a similar input power at $t=0.7 \mathrm{~s}$ for a droop/active power controlled VSC. The deviation in direct voltage response following a change in bus power is clear; notably, there is no oscillatory behaviour during the transition. A comparison of the numerically estimated frequency response to the analytically derived response is shown in Fig. 9. Both responses are a close match, there is no oscillatory behaviour, and the steady-state deviation seen the time-domain response is clear in the frequency response. That is, both droop and active power control impose a steady-state impedance on the system; whereas in the case of direct voltage control, the imposed impedance vanishes in steady-state. In the same manner, Fig. 10 shows the sensitivity of the droop impedance response to variation in operating point across the range of the VSC. Compared to a direct voltage VSC, the phase differs considerably for a droop VSC across the entire range of operation.

To summarize, Fig. 11 shows the terminal-level twoport circuit equivalent of the controlled VSC with the converter local control response behind [33]. This is the equivalent representation of the VSC at the network-level.

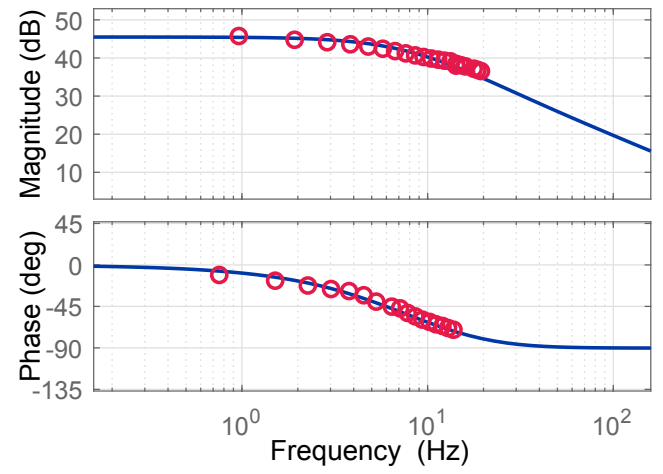

Figure 9: Nominal frequency response of the input-output impedance of a droop/active controlled VSC: analytical (solid blue), numerical estimation (circled red)

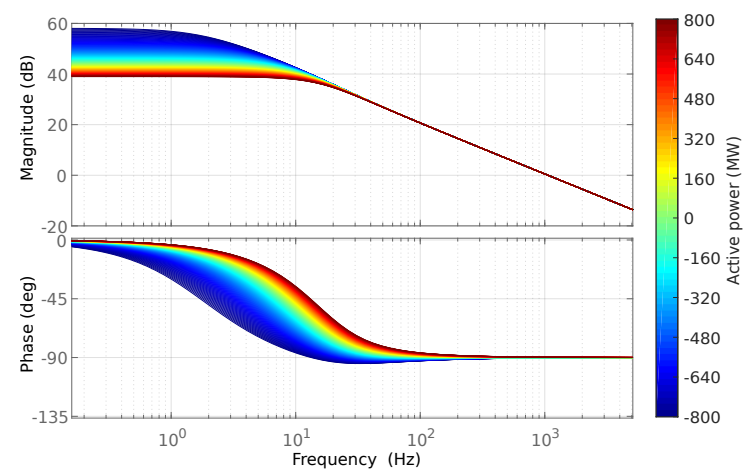

Figure 10: Sensitivity of impedance response to varying operating points

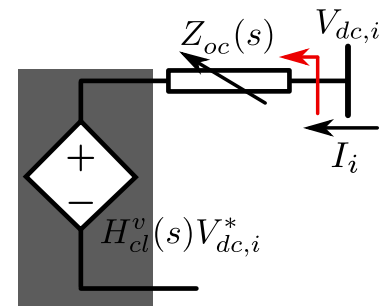

Figure 11: Generic terminal equivalent representation of a controlled VSC

\section{Network Aggregation and Two-partition Equiv- alent for Control Design}

\subsection{Aggregation of Impedance Equivalents}

System-level analysis requires interconnection of different converters with a network and its topology. In the previous modelling section, each VSC has been represented by their equivalent input-output impedances that dictates their external behaviour. Each cable in the network can be represented by its equivalent frequency-dependent transfer function model [35]. In this paper, a distributed $\Pi$ equivalent model utilized. However, higher-order cable models can be integrated as well if a wider range of interaction frequencies are expected.

Subsequent to interconnection in the given topology of the network (with at least two terminals), an equivalent 


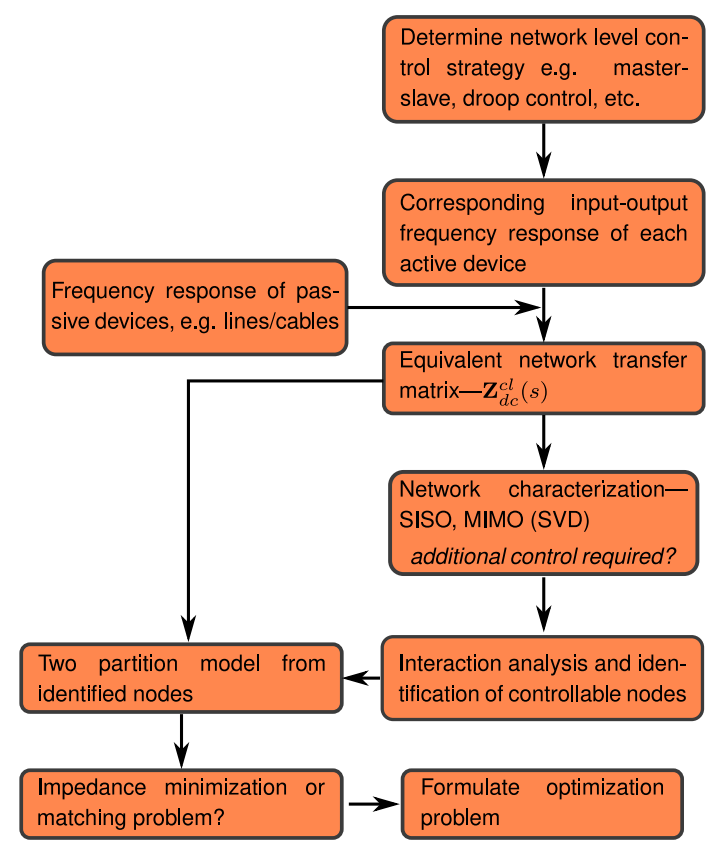

Figure 12: Global control design flowchart

multi-input multi-output (MIMO) dynamic impedance transfer matrix can be obtained as [16]

$$
\underbrace{\left(\begin{array}{c}
\Delta V_{d c, i}^{g l} \\
\Delta V_{d c, j}^{g l} \\
\vdots \\
\Delta V_{d c, n}^{g l}
\end{array}\right)}_{\Delta \mathbf{V}_{d c}^{g l}}=\underbrace{\left(\begin{array}{cccc}
Z_{i i}(s) & Z_{i j}(s) & \cdots & Z_{i n}(s) \\
Z_{j i}(s) & Z_{j j}(s) & \cdots & Z_{j n}(s) \\
\vdots & \vdots & \ddots & \vdots \\
Z_{n i}(s) & Z_{n j}(s) & \cdots & Z_{n n}(s)
\end{array}\right)}_{\mathbf{Z}_{d c}^{c l}(s)} \underbrace{\left(\begin{array}{c}
\Delta I_{i} \\
\Delta I_{j} \\
\vdots \\
\Delta I_{n}
\end{array}\right)}_{\Delta \mathbf{I}}
$$

where $\mathbf{V}_{d c}^{g l}$ is the global direct voltage measurements at each terminal during disturbances, $\Delta \mathbf{I}$ is the vector of bus current changes, and $\mathbf{Z}_{d c}^{c l}(s)$ is the closed-loop dynamic MIMO equivalent transfer matrix of the network. It consists of the contribution of all devices in the networkpassive and active devices as seen from any terminal. Although $\mathbf{Z}_{d c}^{c l}(s)$ is a MIMO matrix, its single-input singleoutput (SISO) responses can be employed to characterize the network as a first step in determining the overall behaviour. However, MIMO responses are more conclusive in determining if the network meets a pre-defined minimum requirement. If not, then interaction analysis may be carried out with a view to implementing global controllers in addition to the existing local controllers.

\subsection{Two-Partition Equivalent Model}

From the perspective of control, manipulating $\mathbf{V}_{d c}^{g l}$ to meet network-level objectives - such as disturbance rejection, requires manipulating $\mathbf{Z}_{d c}^{c l}(s)$. This in turn requires a tractable form of the matrix. One form relies on the input-output stability based on a two-partition model of the network as seen from any controllable terminal. That is, at any controllable terminal $i, \Delta V_{d c, i}^{g l}$ from (8) can be re-written as [16]

$$
\begin{gathered}
\Delta V_{d c, i}^{g l}=\Delta V_{d c, i}+\Delta V_{n e t, i} \\
=Z_{o c, i}(s) \frac{1}{1+\frac{Z_{o c, i}(s)}{Z_{n e t, i}(s)}} \Delta I_{i} \\
Z_{n e t, i}(s)=\frac{Z_{i i}(s) Z_{o c, i}(s)}{Z_{o c, i}(s)-Z_{i i}(s)}
\end{gathered}
$$

where $\Delta V_{d c, i}$ is the local response as derived in Sections 2.1 and 2.2, $\Delta V_{\text {net }, i}$ is the contribution of the rest of the interconnection as seen from terminal $i$, represented by $Z_{n e t, i}(s)$. Since $Z_{o c, i}(s)$ is based on the existing local control, $Z_{\text {net }, i}(s)$ becomes the tractable component which can take on two possible limiting values: $Z_{\text {net }, i} \rightarrow 0$ and $Z_{n e t, i} \rightarrow \infty$. The former is an impedance minimization problem and the latter is an impedance matching problem. The impedance minimization may apply to a case where the local response based on $Z_{o c, i}(s)$ is not acceptableit has one or more poorly damped local oscillatory modes (but strictly stable). Hence, it is desired to minimize these oscillatory modes in addition to any global interaction mode. This article deals with impedance minimization, whereas [36] discusses the case of impedance matching.

Broadly speaking, in both cases the stability margin as seen from each terminal is being manipulated. The generalized steps for analysis and design of global controllers (if required) are depicted in Fig. 12. The optimization formulation is discussed in more detail in later sections.

\section{Application Example: Network Characteriza- tion and Interaction Analysis}

This section presents the first step in determining if additional control action is required. For a three-terminal master-slave controlled HVDC grid shown in Fig. 13, the characterization, and interaction analysis are presented given the local impedance responses derived in Section 2direct voltage and active power. Data for the HVDC grid is presented in Table 1. The HVDC interconnects two AC grid with an isolated power system - such as a wind farm, with VSC-1 being the direct voltage controlling terminal and, VSC-2 and 3 in active power control modes.

Given the network-level control strategy and the corresponding input-output frequency response of all subsystems, the next step is to obtain the network transfer matrix and characterize the network.

\subsection{Equivalent Transfer Matrix and SISO Network Char- acterization}

For the three terminal network shown in Fig. 13 the equivalent transfer matrix can be symbolically written as

$$
\mathbf{Z}_{d c}^{c l}(s)=\left(\begin{array}{ccc}
Z_{11}(s) & Z_{12}(s) & Z_{13}(s) \\
Z_{12}(s) & Z_{22}(s) & Z_{23}(s) \\
Z_{13}(s) & Z_{23}(s) & Z_{33}(s)
\end{array}\right) .
$$




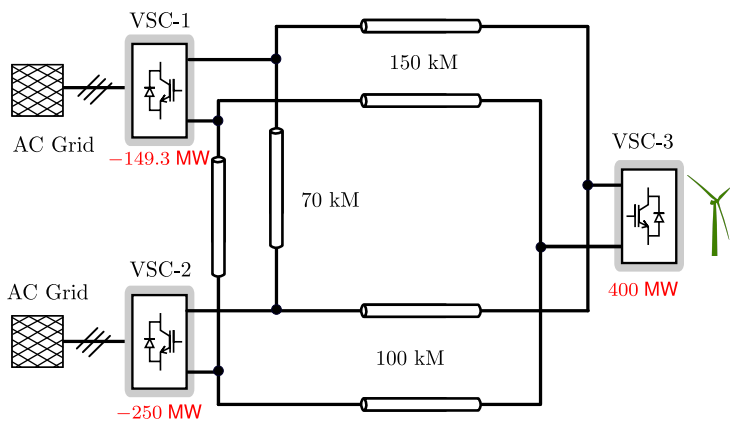

Figure 13: Three terminal VSC-HVDC Grid

Given the nominal power flow, the isolated SISO frequency responses of the network transfer matrix are shown in Fig. 14 (elements in similar colours are equivalent). The diagonal elements predict the behaviour of each subsystem (VSCs) relative to the network for disturbances close to them; whereas off-diagonal elements indicate the transfers from other subsystems - in which case they predict the interactions between terminals. As shown, there appear to be multiple resonant frequencies. There are three dominant interaction frequencies at $17 \mathrm{~Hz}, 41 \mathrm{~Hz}$, and $56 \mathrm{~Hz}$ respectively. However, some elements show all three frequencies with different magnitudes, and others show only two. Hence, the SISO frequency response gives a first impression of potential resonances during disturbances. Each element predicts the dynamic amplification for isolated disturbances from the corresponding terminal or between terminals.

\subsection{MIMO Network Characterization}

The SISO frequency response of $\mathbf{Z}_{d c}^{c l}(s)$ provides rich information on the oscillatory behaviour and resonances as contributed by each VSC. Particularly, the SISO frequency response gives the isolated behavioural responses even though physical responses are often coupled. That is, a disturbance at one VSC elicits a reaction from that VSC and other VSCs that could go back and forth; this is a MIMO behaviour. Essentially, the $\mathbf{Z}_{d c}^{c l}(s)$ transfer matrix should instead be considered as a MIMO transfer matrix rather than a collection of SISO transfer functions. On the other hand, it is important to determine if oscillatory behaviour does indeed warrant additional control action. In this case, the collective response (rather than isolated SISO responses) with respect to defined network specifications indicates the true characterization of the network considering a MIMO behaviour. Specifications can be defined by imposing limits on the maximum singular value of the transfer matrix. For a maximum allowed voltage deviation of $\Delta V$ and expected bus current change of $\Delta I$, a limit can be defined as

$$
\bar{\sigma}\left(\mathbf{Z}_{d c}^{c l}\right) \leq 20 \log _{10} \frac{\|\Delta V\|_{2}}{\|\Delta I\|_{2}} \mathrm{~dB} .
$$

For a maximum allowed voltage deviation of $40 \mathrm{kV}$ (equivalent to $10 \%$ overvoltage), and bus current change of 500
A (equivalent to $200 \mathrm{MW}$ ), the limit of maximum singular value is $\approx 40 \mathrm{~dB}$. Fig. 15 shows the corresponding MIMO responses based on the singular value decomposition (SVD) of the matrix indicating the principal components of responses considering the potential direction of inputs (in this case currents). It shows that the maximum singular value breached the established limits indicated below the grey area. Hence, additional control is required to keep the system within established limits, which may not be possible with existing local control. Therefore, the area below the grey shading in Fig. 15 establishes the benchmark for potential improvements with external controllers.

\subsection{Interaction Analysis}

The SVD response plot still does not provide information about how the VSCs are contributing to each frequency if there is indeed any interaction. While the SVD response may identify a resonant frequency as dominant, this may not hold from an interaction contribution perspective. More so, it is important to isolate the different combinations of terminals contributing to an interaction mode to allow efficient targeting. The frequencydependent relative gain array (RGA) defined for MIMO systems is an extraordinary tool for indication of combinations of interactions in MIMO systems [37]. Let $\mathbf{R}(s)$, evaluated at each frequency denote the corresponding RGA of $\mathbf{Z}_{d c}^{c l}(s)$ such that,

$$
\mathbf{R}(s)=\mathbf{Z}_{d c}^{c l}(s) \otimes\left(\mathbf{Z}_{d c}^{c l}(s)\right)^{-T}
$$

where $\otimes$ is the Hadamard product (element-wise multiplication). Since $\mathbf{R}(s)$ is frequency-dependent, the general criterion that guarantees a non-interacting system at a specified frequency $s=j \omega$ is

$$
\mathbf{R}(j w)=\mathbf{I}
$$

where $\mathbf{I}$ is the identity matrix. That is, VSCs are not interacting at a specified frequency if $\mathbf{R}(s)$ is unity. On the other hand, they are interacting at a specified frequency if corresponding elements in $\mathbf{Z}_{d c}^{c l}(s)$ show magnitude peaks $(\gg 1)$. For example, if all elements of $\mathbf{Z}_{d c}^{c l}(s)$ show magnitude peaks at a certain frequency, then all VSCs are interacting at that frequency. Additionally, RGA also allows determining how inherent coordination could be applied in design when the number of controllers to be designed is less than the number of VSCs in the network.

For the studied example, Fig. 16 shows the frequencydependent RGA magnitude plot based on the network transfer matrix $\mathbf{Z}_{d c}^{c l}(s)$. The combination of terminals contributing to each interaction frequency is shown in the zoomed response. Several observations can be directly made: Observe the first peak where all distinct elements of the matrix show significant peaks; at this frequency, all VSCs are interacting or otherwise contributing to that frequency at different magnitudes. This is due to the interaction between the direct voltage control and the total 

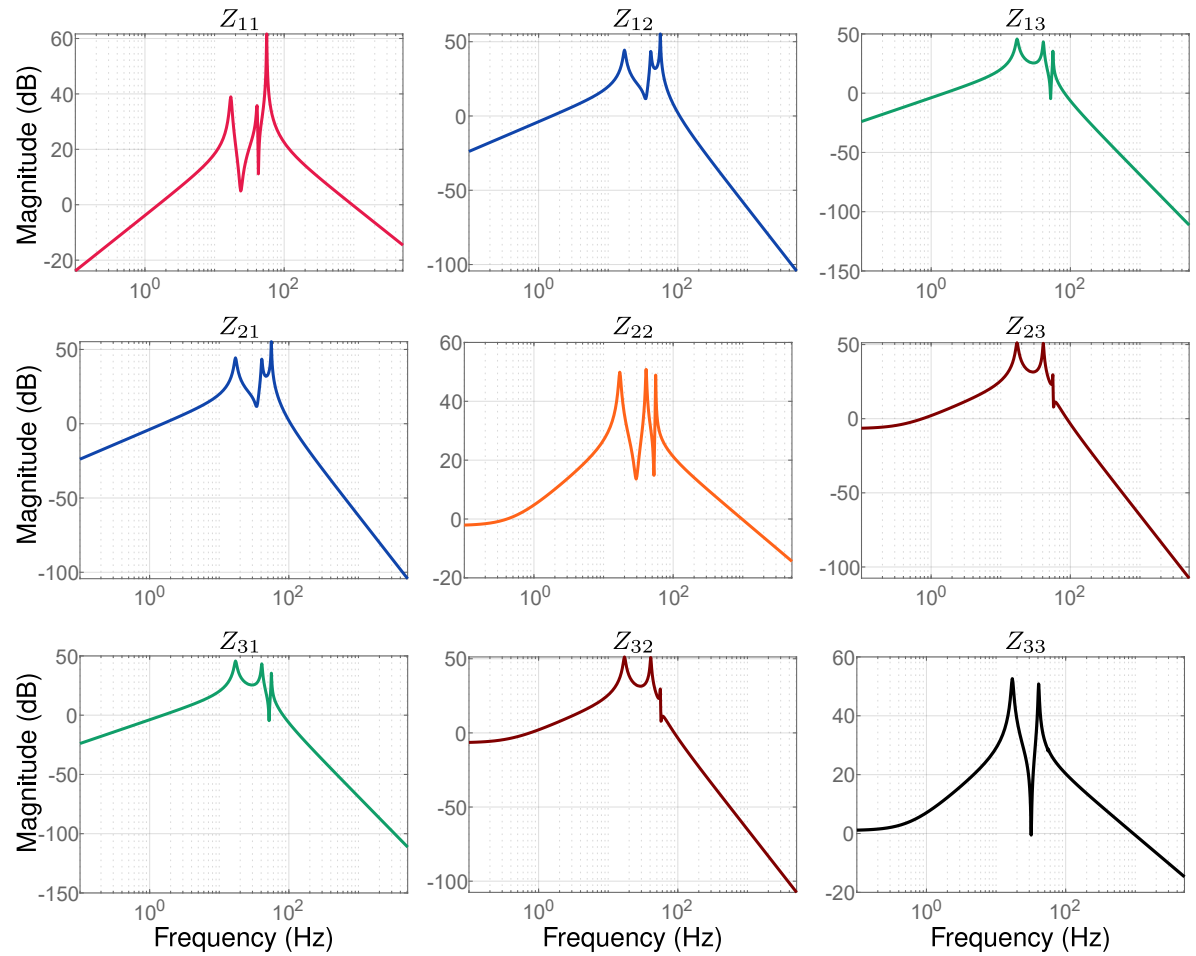

Figure 14: SISO frequency response of the global disturbance transfer matrix

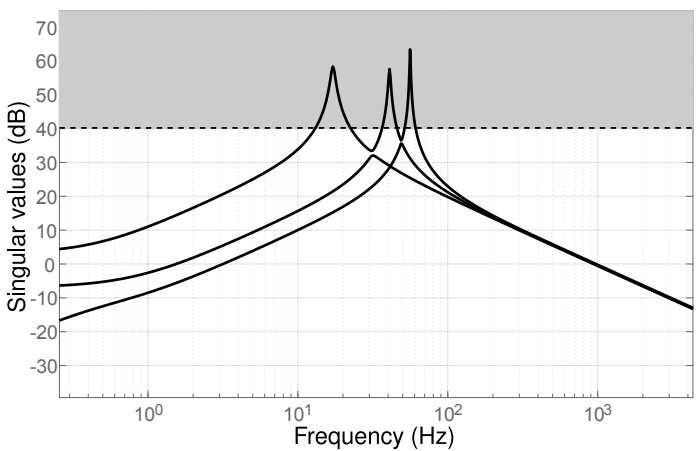

Figure 15: SVD plot of the global closed-loop disturbance response matrix

capacitance of the DC grid which was not considered in local control. Nevertheless, observation of the first peak is contrary to the SVD plot in Fig. 15 that suggest the most dominant mode as the resonance at $56 \mathrm{~Hz}$ (third peak). At the second peak, VSCs 1 and 2 are interacting with VSC-3 as indicated by the magnitudes of 13,23 , and 33 elements. Whereas, at the slightly higher third peak, only VSC-1 and 2 are interacting with a relatively higher magnitude than the previous peak (VSC-3 doesn't seem to participate at all this frequency). Further, at steady-state and high frequency, diagonal elements approach 1 and offdiagonal elements approach 0-non-interacting system in these spectra. From this plot, it can be hypothesized that only two controllers at VSC- 1 and 2 are necessary to decouple the system. It makes no sense to have three controllers since for each frequency VSC-3 is contributing, either one

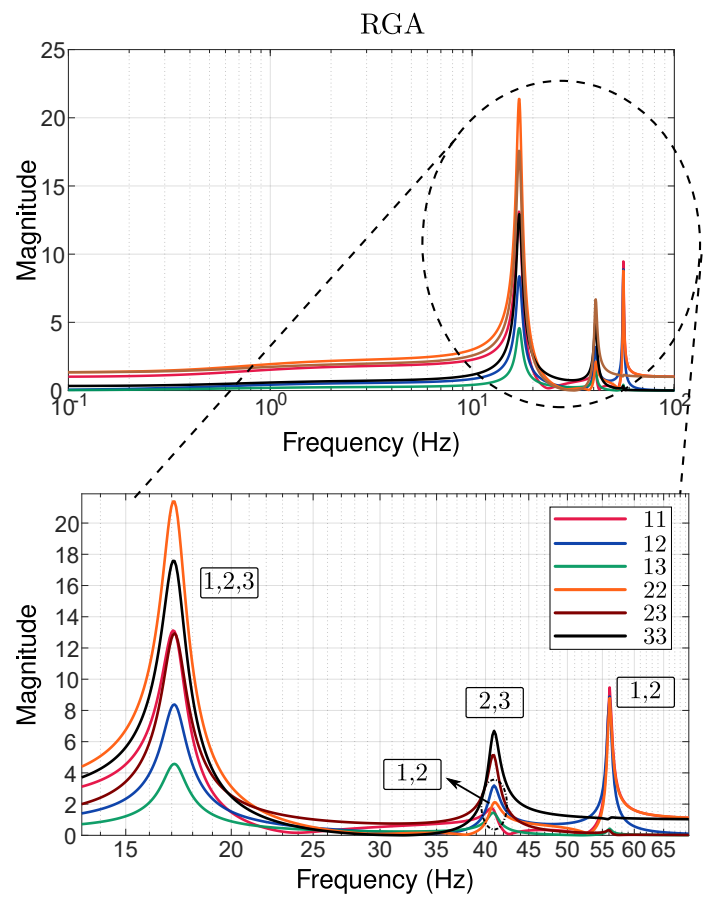

Figure 16: Frequency-dependent RGA plot

of VSC-1 and/or VSC-2 are also contributing.

To verify these frequencies from a detailed nonlinear time-domain model, Fig. 17 depicts the time-domain responses of direct voltages for a step change at $2.5 \mathrm{~s}$ at VSC3 and the corresponding fast Fourier transform (FFT). Firstly, the frequencies of resonances match the identified 


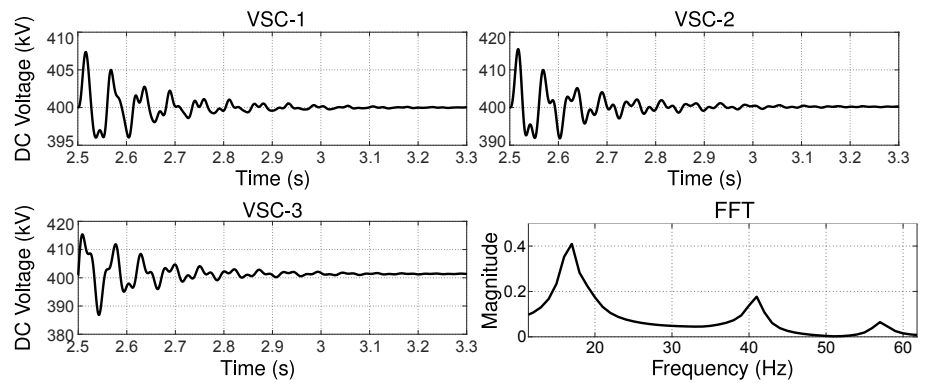

Figure 17: Non-linear time-domain simulation of system responses and FFT of signals for step change in power from VSC-3
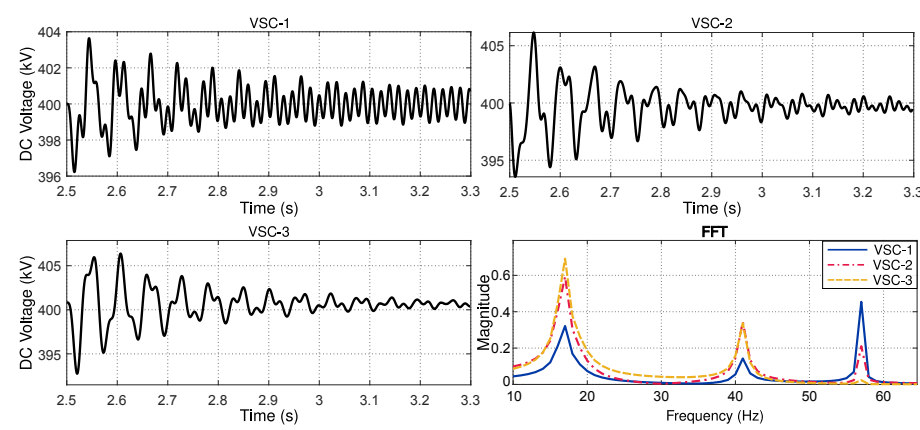

Figure 18: Non-linear time-domain simulation of system responses and FFT of signals for step change in power at VSC-2

frequencies in Fig. 15 at $17 \mathrm{~Hz}, 41 \mathrm{~Hz}$, and $56 \mathrm{~Hz}$ respectively. Secondly, for the change in VSC-3, the first two frequencies are more dominant as expected from the interaction analysis since the contribution of VSC-3 to the 56 $\mathrm{Hz}$ resonance is low. To further demonstrate the effectiveness of the RGA plot in the detection of interactions, Fig. 18 depicts the time-domain response of direct voltages for a step-change in power reference at VSC-2, with the corresponding FFT of signals. From the RGA response, for changes at VSC-2, VSC-1 contributes significantly to the first and third peaks; this is confirmed by the FFT with relatively similar magnitudes to the RGA response. At VSC-2 all three peaks can be seen and at VSC-3 only the first and second peaks can be seen since it does not contribute to the third peak that only involves VSC-1 and 2 .

\section{Application Example: Control Formulation and Design of Decentralized Global Controllers}

The network analysis in the previous section established the need to implement global controllers independent of the existing local control. The next step from Fig. 12 is to partition the system into two from each controllable terminal according to (9). In this case, an impedance minimization problem is desired due to the presence of a poorly damped local oscillatory mode in the direct voltagecontrolled VSC. That is, it is desired to transfer some local responsibilities to the global controller, in addition to mitigating interactions with the rest of the network. Hence,

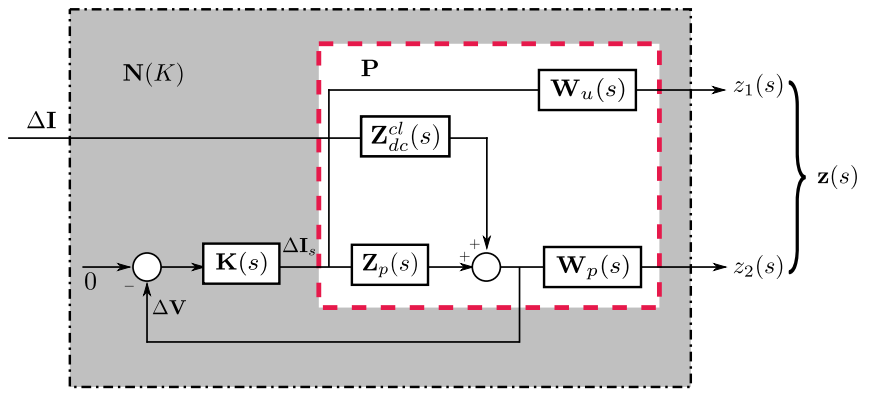

Figure 19: Block diagram of plant interconnections

it is desired that $Z_{n e t, i} \rightarrow 0$ and according to (10) this is equivalent to forcing $Z_{i i}(s) \rightarrow 0$ from each terminal since there is no access to local control based on $Z_{o c, i}(s)$. Therefore, the goal of global control from the network view is to minimize the existing transfer matrix $\mathbf{Z}_{d c}^{c l}(s)$ subject to constraints.

\subsection{Objectives of Design}

First, the objectives are stated, and a description of how the problem fits into the $\mathcal{H}_{\infty}$ mixed sensitivity framework follows. It is important to remark again that this is a disturbance rejection problem. The following objectives are paramount:

1. Reduce the effect of feedback coupling (interaction) between terminals and dormant network oscillations not considered during the individual design of local controllers.

2. Synthesized controllers must not interact with local controllers. Particularly, it is ideally desired that synthesized controllers vanish in steady-state. That is, the controllers are only active during transients and disturbances of pre-defined frequency spectrum identified based on $\mathbf{Z}_{d c}^{c l}(s)$.

3. More importantly, the controller must not in any manner interact with the inner AC control loop which is often the fastest loop and the bandwidth limit of the VSC.

In the following subsections, these objectives are translated into frequency domain requirements.

\subsection{Mixed Sensitivity Framework}

Considering that tracking is not an issue as each local controller ensures this, the problem becomes that of minimizing $\Delta \mathbf{V}_{d c}^{g l}=\mathbf{Z}_{d c}^{c l}(s) \Delta \mathbf{I}$ due to the interconnection with other VSCs, subject to constraints. Constraints include the maximum allowed control effort to minimize $\Delta \mathbf{V}_{d c}^{g l}$. Therefore, the overall problem is a mixed-sensitivity problem of disturbance and control input.

Fig. 19 describes the problem in the generalized framework of $\mathcal{H}_{\infty}$ [38]. $\mathbf{Z}_{p}=\alpha I$ is a fictitious plant model ( $\alpha$ is determined by iteration and to prevent the synthesis of a 
large gain controller); in this work, $\alpha=[1-500]$. This ensures that the minimization problem is wholly dominated by $\mathbf{Z}_{d c}^{c l}(s)$. $\mathbf{W}_{p}(s)$ is the output weighting matrix that determines by how much $\Delta \mathbf{V}_{d c}^{g l}$ is minimized (objective 1), $\mathbf{W}_{u}(s)$ is the control output weighting matrix (objectives 2 and 3$)$, and $\mathbf{K}(s)$ is a diagonal matrix of synthesized controllers [39]. It must be noted that this is a MIMO problem and all matrices are MIMO matrices with lengths equal to the number of subsystems. The closed-loop expressions of the augmented system can be derived from Fig. 19. Starting with the inner-most structure (dashed red box)

$$
\begin{aligned}
z_{1}(s) & =\mathbf{W}_{u}(s) \Delta \mathbf{I}_{s} \\
z_{2}(s) & =\mathbf{W}_{p}(s) \mathbf{Z}_{d c}^{c l}(s) \Delta \mathbf{I}+\mathbf{W}_{p}(s) \mathbf{Z}_{p}(s) \Delta \mathbf{I}_{s} \\
\Delta \mathbf{V} & =\mathbf{Z}_{d c}^{c l}(s) \Delta \mathbf{I}+\mathbf{Z}_{p}(s) \Delta \mathbf{I}_{s} .
\end{aligned}
$$

The above expressions can be written compactly as

$$
\left[\begin{array}{c}
\mathbf{z} \\
\Delta \mathbf{V}
\end{array}\right]=\underbrace{\left[\begin{array}{ll}
P_{11} & P_{12} \\
P_{21} & P_{22}
\end{array}\right]}_{\mathbf{P}}\left[\begin{array}{c}
\Delta \mathbf{I} \\
\Delta \mathbf{I}_{s}
\end{array}\right], \quad \mathbf{z}=\left[\begin{array}{l}
z_{1} \\
z_{2}
\end{array}\right]
$$

where $\mathbf{P}$ is the augmented plant with target weights $\mathbf{W}_{p}$ and $\mathbf{W}_{u}, z_{1}$ and $z_{2}$ are the outputs to be minimized, $\Delta \mathbf{V}$ is the input to controller, $\Delta \mathbf{I}$ is the disturbance, and $\Delta \mathbf{I}_{s}$ as the control input. The (s) term for transfer functions is henceforth neglected. The $\mathbf{P}$ matrix can be expanded as

$$
\mathbf{P}=\left[\begin{array}{c|c}
0 & \mathbf{W}_{u} \\
\mathbf{W}_{p} \mathbf{Z}_{d c}^{c l} & \mathbf{W}_{p} \mathbf{Z}_{p} \\
\hline \mathbf{Z}_{d c}^{c l} & \mathbf{Z}_{p}
\end{array}\right]
$$

After interconnection with the controller(s) to be synthesized, then the controller outputs $\Delta \mathbf{I}_{s}=-\mathbf{K} \Delta \mathbf{V}$ can be eliminated to obtain

$$
\begin{aligned}
& z_{1}=-\mathbf{W}_{u} \mathbf{K} \Delta \mathbf{V} \\
& z_{2}=\mathbf{W}_{p} \mathbf{Z}_{d c}^{c l} \Delta \mathbf{I}-\mathbf{W}_{p} \mathbf{Z}_{p} \mathbf{K} \Delta \mathbf{V} \\
& \Delta \mathbf{V}=\mathbf{Z}_{d c}^{c l} \Delta \mathbf{I}-\mathbf{Z}_{p} \mathbf{K} \Delta \mathbf{V} \\
& \Longrightarrow \Delta \mathbf{V}=\underbrace{\frac{\mathbf{I}}{\mathbf{I}+\mathbf{Z}_{p} \mathbf{K}}}_{\mathbf{S}} \mathbf{Z}_{d c}^{c l} \Delta \mathbf{I} \\
& \mathbf{Z}_{d c, \text { new }}^{c l}
\end{aligned}
$$

where $\mathbf{S}$ is the sensitivity transfer matrix of the synthesized controllers, $\mathbf{Z}_{d c, n e w}^{c l}$ is the modified transfer matrix of the network, and $\Delta \mathbf{V}$ can be eliminated from $z_{1}$ and $z_{2}$ to obtain

$$
\begin{aligned}
& z_{1}=-\mathbf{W}_{u} \mathbf{K S Z}_{d c}^{c l} \Delta \mathbf{I} \\
& z_{2}=\mathbf{W}_{p} \mathbf{S Z}_{d c}^{c l} \Delta \mathbf{I} .
\end{aligned}
$$

The above expressions can be written compactly as

$$
\mathbf{z}=\mathbf{N}(\mathbf{K}) \Delta \mathbf{I}
$$

The optimization problem becomes that of minimizing the $\mathcal{H}_{\infty}$ norm of the closed loop transfer function $\mathbf{N}$ subject to a given stable controller such that

$$
\min _{K}\|\mathbf{N}(K)\|_{\infty} \leq \gamma, \quad \mathbf{N}(K)=\left[\begin{array}{c}
\mathbf{W}_{u} \mathbf{K S G}_{d} \\
\mathbf{W}_{p} \mathbf{S G}_{d}
\end{array}\right]
$$

where $\gamma$ is the desired closed-loop gain.

\subsection{Design Preliminaries}

\subsubsection{Scaling}

A key priority for a successful implementation is proper scaling to prevent potential skewing of the mixedsensitivity problem. Scaling is done according to the recommendations in [38] by determination of maximum expected change in inputs, maximum allowed control input to correct output error, and maximum allowed output for each subsystem. Scalings at each subsystem can emphasize the priority or role of each subsystem. For example, a maximum of $20 \mathrm{kV}$ may be allowed at one subsystem, while only $10 \mathrm{kV}$ may be allowed at another subsystem. However, for simplicity in this paper, the scaling is similar at all subsystems as given in Table 3. The fictitious plant model $\mathbf{Z}_{p}$ and transfer matrix $\mathbf{Z}_{d c}^{c l}$ are scaled according to

$$
\begin{array}{r}
\mathbf{Z}_{p}^{s}=\Delta \mathbf{V}_{\text {max }}^{-1} \mathbf{Z}_{p} \Delta \mathbf{I}_{s, \max } \\
\mathbf{Z}_{d c}^{c l, s}=\Delta \mathbf{V}_{\text {max }}^{-1} \mathbf{Z}_{d c}^{c l} \Delta \mathbf{I}_{\max }
\end{array}
$$

where $\mathbf{I}_{s, \max }$ is the maximum allowed control input to minimize output, and $\mathbf{I}_{\max }$ is the maximum expected disturbance.

Table 3: Scaling Factors

\begin{tabular}{ccc}
\hline \hline Parameter & Value & Comments \\
\hline$\Delta V_{\max }$ & $40 \mathrm{kV}$ & allowed voltage deviation \\
$\Delta I_{s, \max }$ & $0.8 \mathrm{kA}$ & allowed control input \\
$\Delta I_{\max }$ & $0.5 \mathrm{kA}$ & expected disturbance \\
\hline
\end{tabular}

\subsubsection{Model Reduction}

To keep the problem as tractable as possible by preventing numerical artefacts, a low order equivalent model is sought for the key transfer matrix $\mathbf{Z}_{d c}^{c l}$. The Hankel balanced reduction method is applied for reduction. In this paper, the total order of $\mathbf{Z}_{d c}^{c l}$ is 35 before reduction and 7 after reduction. Fig. 20 shows the comparison of the original model and the equivalent reduced model, whereas, Fig. 21 depicts the Hankel's singular value plot showing the dominant states.

\subsubsection{Order and Structure of Controller}

A key priority is to obtain adoptable low-order fixed structure controllers. It is well known that the order of synthesized controller with standard $\mathcal{H}_{\infty}$ is quite high while assuming a centralized structure. Several methods to 

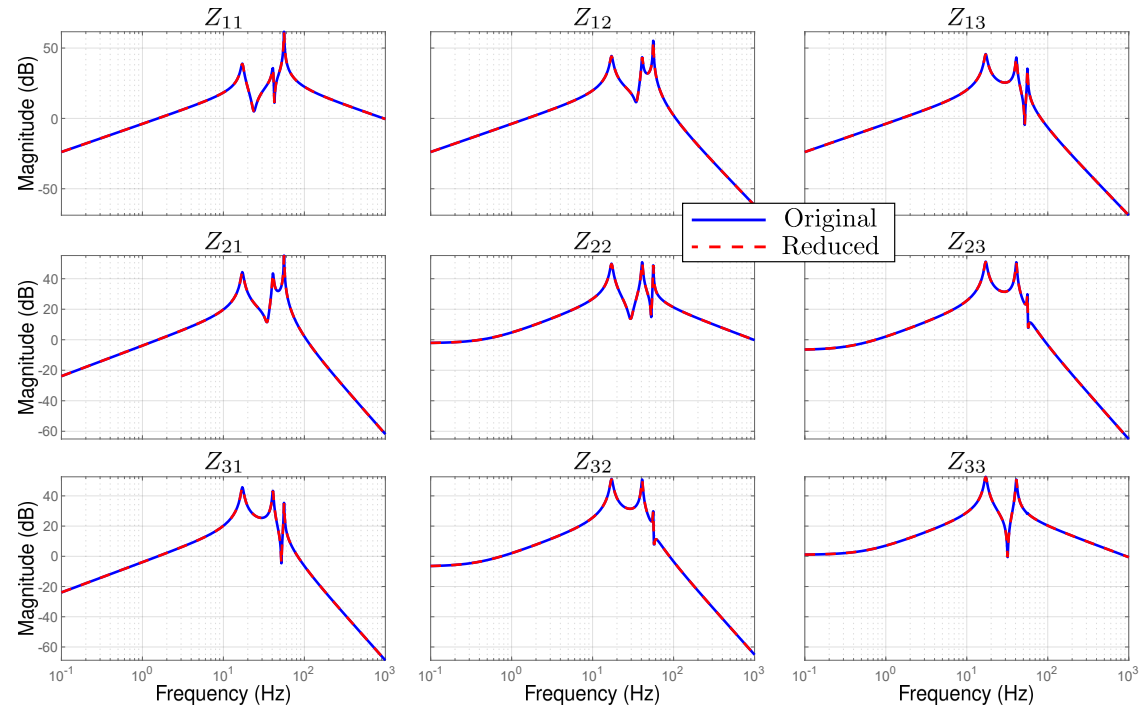

Figure 20: Comparison plot between original and reduced disturbance model

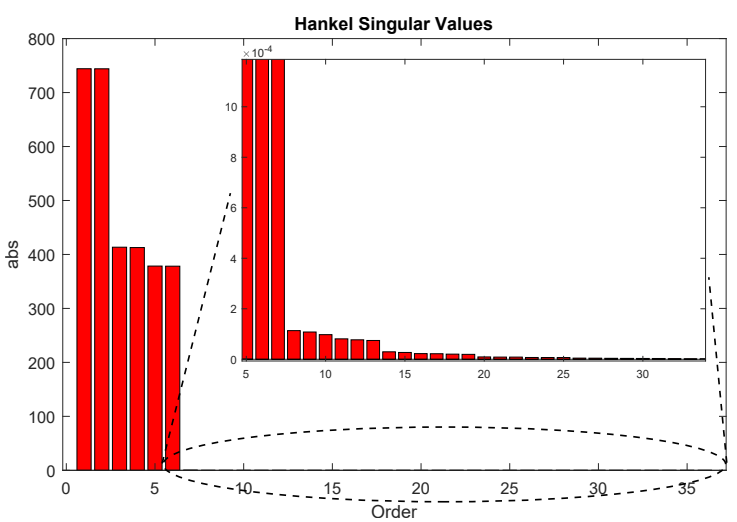

Figure 21: Hankel singular value plot

Table 4: Design Weights

\begin{tabular}{ccc}
\hline \hline & $W_{p}$ & $W_{u}$ \\
\hline VSC-1 & $0.5 \frac{s+2 \omega_{p 1}}{s+\frac{\omega_{p 1}}{500}}$ & $0.75 \frac{s+2}{s+\omega_{u 1}}$ \\
VSC-2 & $0.45 \frac{s+2 \omega_{p 2}}{s+\frac{\omega_{p 2}}{450}}$ & $0.65 \frac{s}{s+\omega_{u 2}}$ \\
VSC-3 & $0.45 \frac{s+2 \omega_{p 3}}{s+\frac{\omega_{p 3}}{450}}$ & $0.65 \frac{s}{s+\omega_{u 3}}$ \\
\hline
\end{tabular}

reduce the order have been suggested in literature. However, in this paper, decentralized controllers, with a maximum order of three was preferred for transparency and to eliminate any communication requirements of the centralized structure. The realized third-order structure is reducible to two without any change in performance if desired.

\subsubsection{Weight Selection and justification}

The choice of weights $\mathbf{W}_{p}$ and $\mathbf{W}_{u}$ are influenced by the actual frequency domain responses, and iteration may be required to obtain the best weights.
- $\mathbf{W}_{p}$ is a diagonal matrix of frequency-dependent weights that determine the target sensitivity of control. For the impedance minimization problem, a simple choice of a high gain low-pass filter with a bandwidth equal to the frequency of disturbances to be rejected can be selected. Bandwidth and magnitude of weights can be chosen to reflect the priority of each subsystem or coordination as obtained from the RGA plot. The chosen weights for the base case can be found in Table 4 with a bandwidth of $\omega_{p 1}=\omega_{p 2}=\omega_{p 3}=2 \pi \times 65(\mathrm{rad} / \mathrm{s})$ respectively for each subsystem.

- $\mathbf{W}_{u}$ is also a diagonal matrix of frequency-dependent weights to constrain the controller action. The weights in $\mathbf{W}_{u}$, in general, are high pass filters. The chosen bandwidth of weights should limit the bandwidth of control to be less than that of the inner current loop (based on generic knowledge) and slightly higher than the disturbance (but not necessarily). Each decentralized controller can have different bandwidths to prioritize and force coordination among them. Table 4 list the weights and corresponding bandwidths of $\omega_{u 1}=2 \pi \times 90$, $\omega_{u 2}=2 \pi \times 75, \omega_{u 3}=2 \pi \times 60(\mathrm{rad} / \mathrm{s})$ respectively. Subsystem 1 is given a slight priority (being the master terminal) by having a slightly higher gain in the low-frequency region.

\subsection{Proposed Decentralized Controller Design}

Decentralized controllers of fixed order are synthesized by solving the $\mathcal{H}_{\infty}$ optimization problem of (21) subject to a stabilizing controller with $\gamma \leq 1$. The control design is implemented with MATLAB ${ }^{\circledR}$ 's hinfstruct algorithm [39]. The pseudocode shown in Algorithm 1 depicts an overview of the controller synthesis procedure as implemented. The order of each controller is fixed at three. Af- 


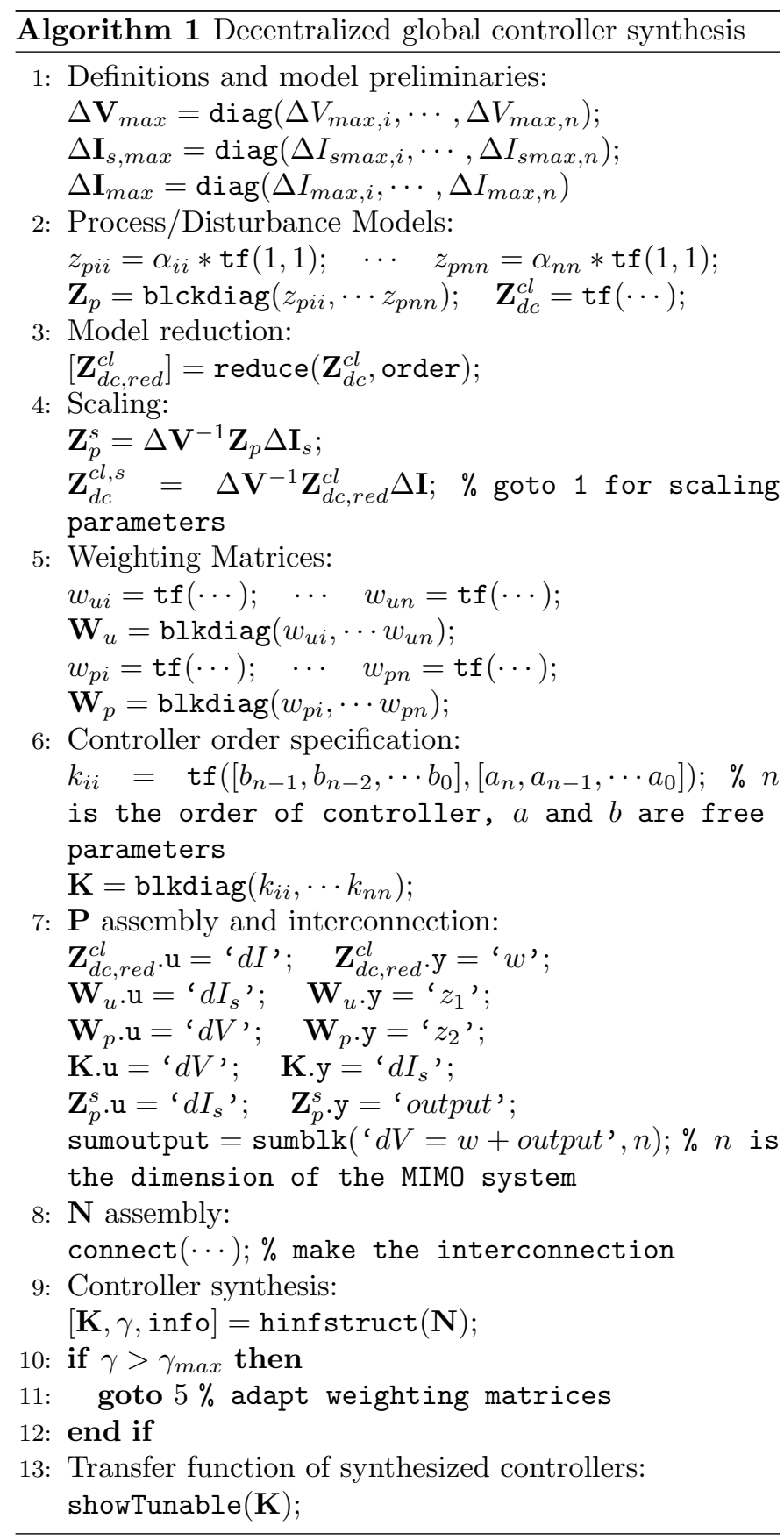

ter the analysis in the previous section, only two controllers are required to decouple the system. Moreover, VSC-3 integrates an intermittent source and may not be suitable as a controllable terminal. Nevertheless, both two and three decentralized controllers are synthesized while including information about the entire grid. In the physical structure the grid, the global controllers accept bus voltage deviations as inputs and outputs current signals to mitigate interaction oscillations as shown in Fig. 22. Therefore, the global controllers are additional feed-forward terms external to the local control. In addition, the global controllers reduce the initial magnitude of voltage deviations at the instant of disturbances.

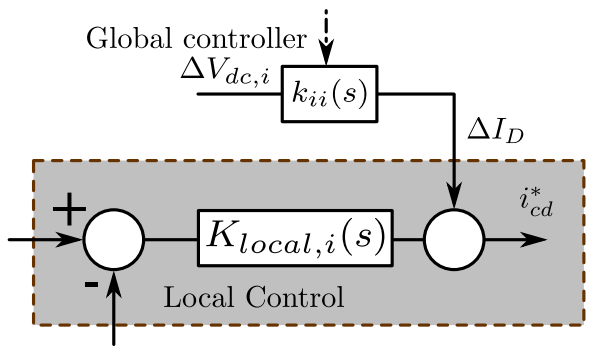

Figure 22: Block diagram of the modified control structure

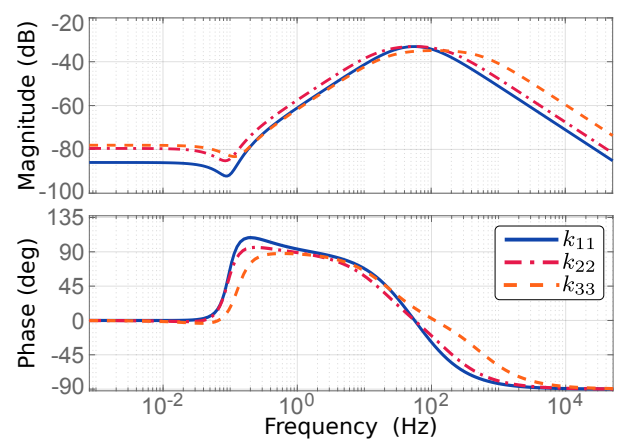

Figure 23: Frequency responses of the three fixed order controllers

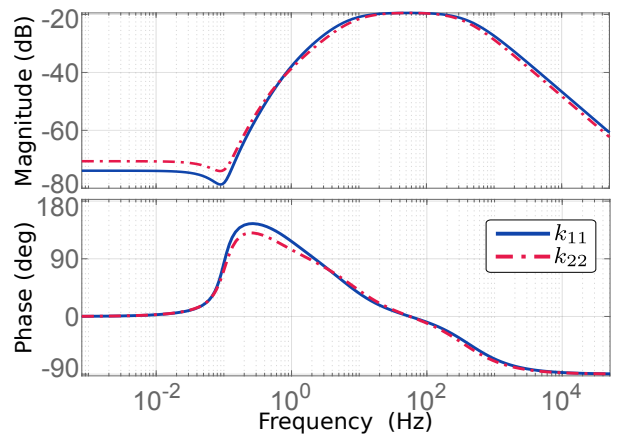

Figure 24: Frequency responses of the two fixed order controllers

For the case of three synthesized controllers, the maximum gain achieved for $\gamma\left(\|\mathbf{N}\|_{\infty}\right)=0.655$ which is less than the target $\gamma_{\max }$. Fig. 23 shows the frequency response of the three synthesized controllers. For this case, VSC-2 and VSC-3 are prioritized equally. However, this is arbitrary and flexibility can be employed with specifics. The synthesized controllers are bandpass filters with active regions around the specified range of frequencies, and very low gain outside this range. As expected, the controllers do not contribute to steady-state behaviour $(<1$ $\mathrm{Hz}$ ) where local controllers are most active. For the case of two controllers designed for VSC-1 and 2, while including the model of the network including VSC-3, the maximum gain achieved for $\gamma\left(\|\mathbf{N}\|_{\infty}\right)=0.9$ which is also less than the target. Fig. 24 shows the frequency response. Note that the magnitude of response is nearly a decade higher to compensate for the lack of a controller at VSC-3.

Based on two decentralized controllers, Fig. 25 shows the modified SISO response of the network transfer matrix 


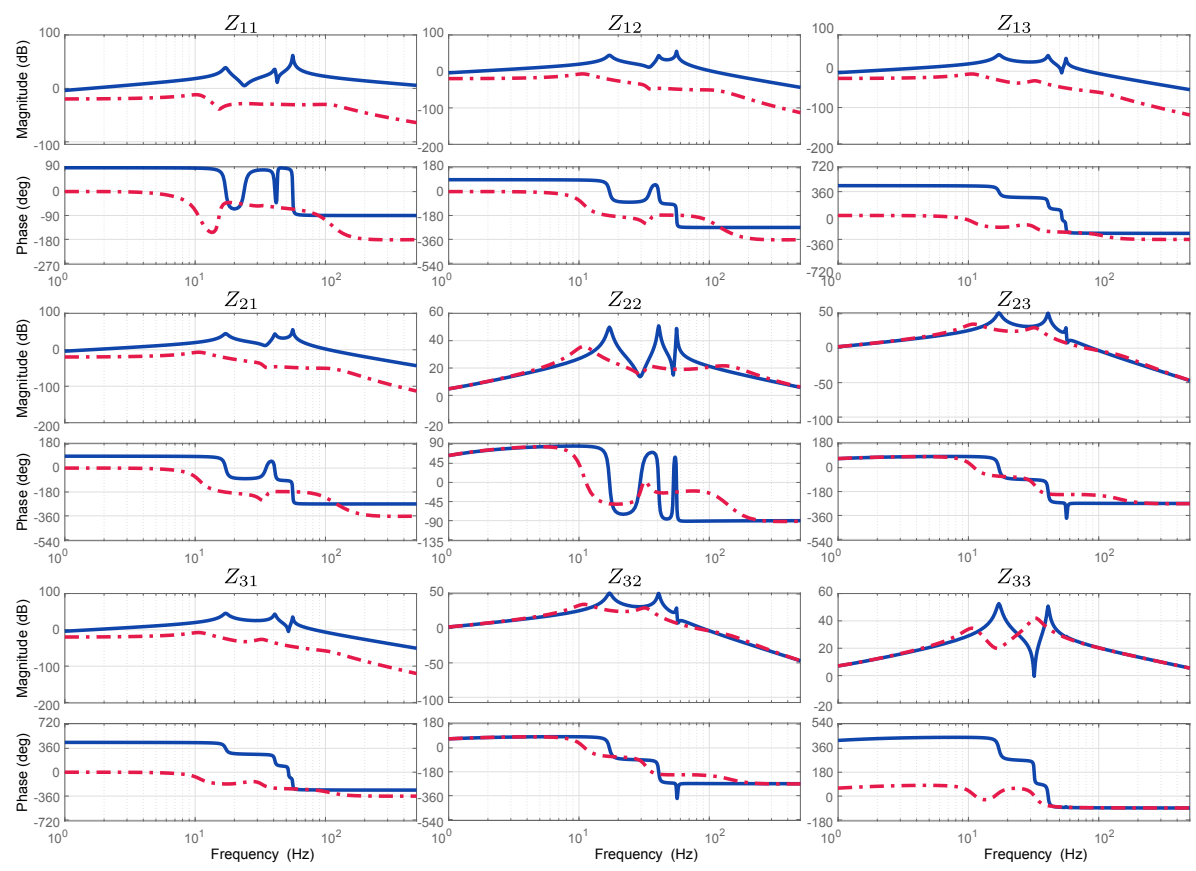

Figure 25: SISO frequency responses: existing local control (bold blue), global control (dash dotted red)

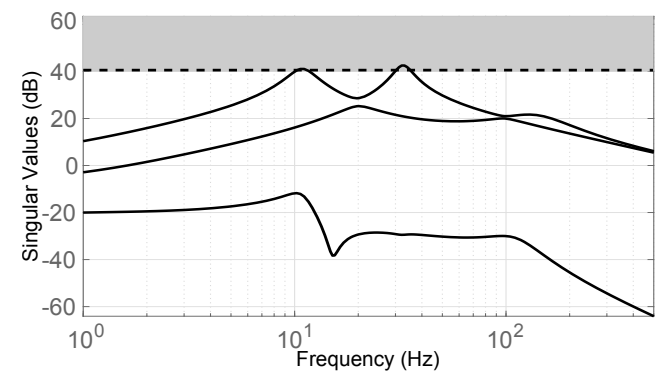

Figure 26: SVD MIMO frequency response with global controllers

in comparison to that with only local controllers. Remarkable improvements can be seen in both magnitude and phase difference. Most importantly, the peaks have been shaved despite having implemented only two controllers. The new network characterization based on MIMO responses is shown in Fig. 26. It shows that the maximum singular value is within the pre-defined limits and the smallest singular value is several orders lower than that in Fig. 15. To establish the modified interaction profile, Fig. 27 shows the modified frequency-dependent RGA. It can be seen that the system has been decoupled in nearly the entire range of disturbance spectrum as diagonal elements approach 1 and off-diagonal elements approach 0. However, a slight interaction between VSC- 2 and 3 can be seen around $10 \mathrm{~Hz}$, but the gain is significantly lower compared to the original system without the global controllers.

\section{Validation and Time-Domain Results}

This section presents time-domain results with the developed global controllers for both cases of two and three

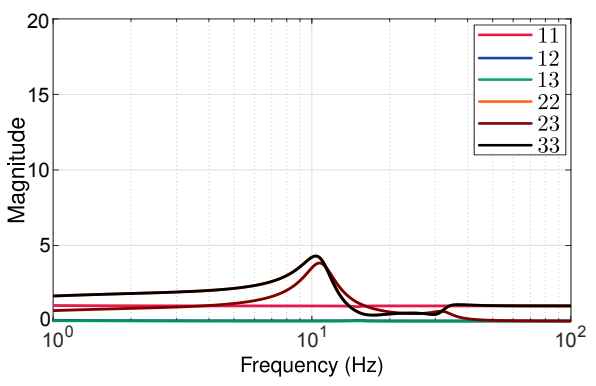

Figure 27: Frequency-dependent relative gain array with supplementary control

decentralized controllers. For the first analysed case in Fig. 17 with a $50 \%$ sudden change in active power at VSC-3, Fig. 28 shows the comparison of direct voltage responses with two global controllers and with only local control. As can be seen, the global controllers were able to quickly damp out the oscillations and stabilize grid parameters within a short time frame. In the second case of Fig. 18 for a similar step-change in active power at VSC2, Fig. 29 shows the grid direct voltage responses. It can be seen that despite the higher distortion across several frequencies as in case with only local control, the global controllers are able to quickly mitigate the oscillatory behaviour. The only oscillatory behaviour is that due to the interaction between VSC-2 and VSC-3 at $10.3 \mathrm{~Hz}$ as shown in the RGA in Fig. 27. However, this damps out within $200 \mathrm{~ms}$.

In a severe case of sudden simultaneous changes at both VSC-2 and 3 ( $50 \%$ and $30 \%$ steps changes respectively), Fig. 30 shows a comparison between only local control and two global controllers. Despite a lack of global con- 

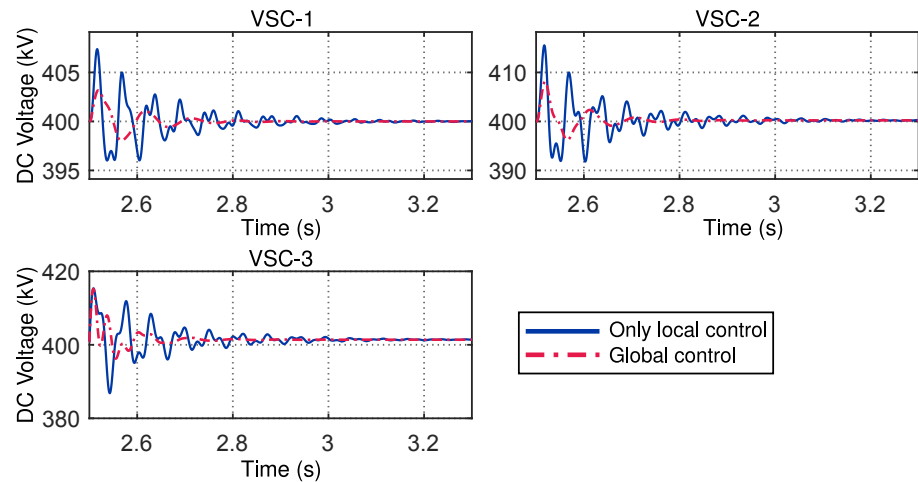

Figure 28: Performance of two synthesized controllers in comparison to case without global controllers for changes at VSC-3
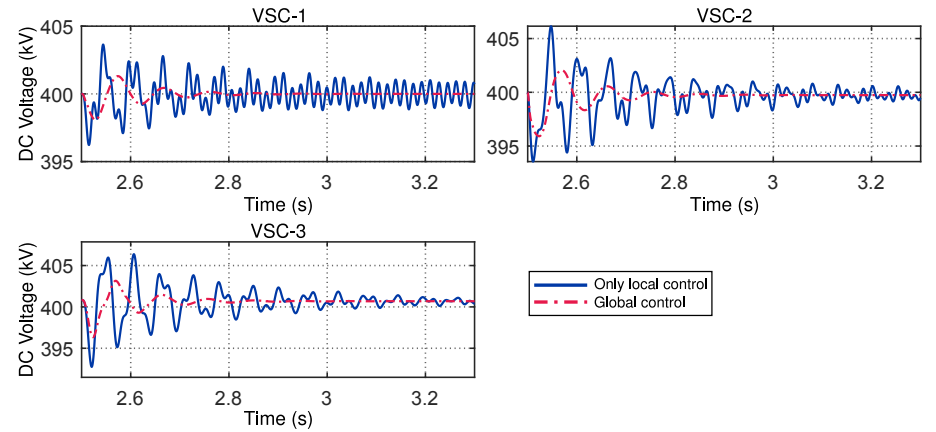

Figure 29: Performance of two synthesized controllers in comparison to case without global controllers for changes at VSC-2

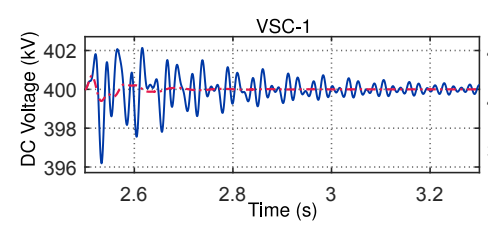

VSC-3

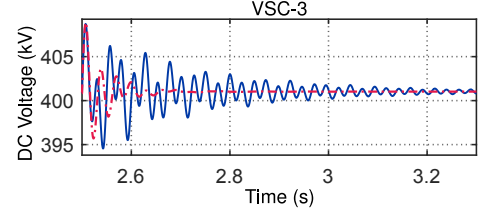

Figure 30: Performance of two synthesized controllers in comparison to case without global controllers for multiple simultaneous changes

troller at VSC-3 at a cost of a slightly degraded response, similar performance as in previous cases demonstrate the improvements from the global control.

To further demonstrate the robustness of synthesized controllers (two decentralized) in a more stringent scenario, Fig. 31 depicts the direct voltage responses across varying operating points. It can be seen that in the case with only local control, the system lost after a sudden disconnection of VSC-3 initially supplying power into the network. However, with the two synthesized global controllers, the network maintained its robust stability facilitated by the controllers, while mitigating detrimental oscillations. As expected for VSC-3, the response is slightly degraded. Nevertheless, the performance is quite better

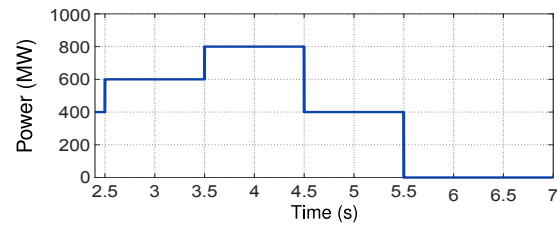

(a)
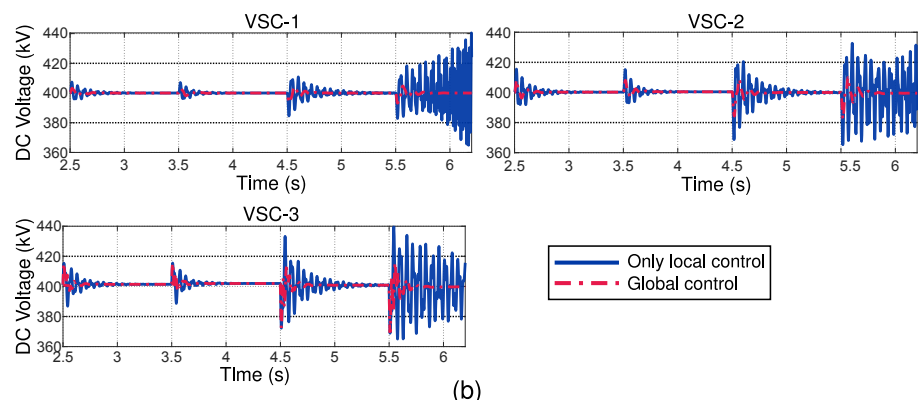

Figure 31: Test of robustness (a) step disturbances at VSC-3 (b) direct voltage response of the network

and acceptable than with only local control.

\section{Conclusion}

In summary, this paper proposed the formulation and design of decentralized global controllers to decouple converters in a VSC-HVDC grids through network impedance shaping. First, it was determined if the characterization of the network through MIMO treatment meets pre-defined minimum requirements. In the case the network does not meet this requirement, then RGA can be applied to identify cross-interactions between VSCs and the mechanism of these interactions. Then, robust controllers are synthesized by fitting the problem into an $\mathcal{H}_{\infty}$ convex optimization framework. Importantly, these controllers can be designed and implemented using only measurable input-out impedances of VSCs and the availability of an additional control channel. Overall, it can be seen that despite the order of the system, the designed low order controllers were able to robustly decouple the system as demonstrated. Therefore, the method presented offer network operators a flexible and scalable approach in securely interconnecting independently designed converters.

\section{Acknowledgements}

This work was financially supported by the European Union's Horizon 2020 research and innovation programme under Marie-Sklodowska-Curie action INCITE (grant agreement No. 675318).

\section{Conflict of Interest}

None declared. 


\section{References}

[1] B. K. Bose, Global energy scenario and impact of power electronics in 21st century, IEEE Transactions on Industrial Electronics 60 (7) (2013) 2638-2651. doi:10.1109/TIE.2012. 2203771.

[2] N. Flourentzou, V. G. Agelidis, G. D. Demetriades, Vsc-based hvdc power transmission systems: An overview, IEEE Transactions on Power Electronics 24 (3) (2009) 592-602. doi: 10.1109/TPEL . 2008. 2008441.

[3] F. Schettler, H. Huang, N. Christl, HVDC transmission systems using voltage sourced converters design and applications, in: 2000 Power Engineering Society Summer Meeting (Cat. No.00CH37134), Vol. 2, 2000, pp. 715-720 vol. 2. doi:10.1109/ PESS. 2000.867439.

[4] D. V. Hertem, M. Ghandhari, Multi-terminal VSC HVDC for the european supergrid: Obstacles, Renewable and Sustainable Energy Reviews 14 (9) (2010) 3156 - 3163. doi:http: //dx.doi.org/10.1016/j.rser.2010.07.068.

URL http://www.sciencedirect.com/science/article/pii/ $\mathrm{S} 1364032110002480$

[5] T. Haileselassie, K. Uhlen, Power system security in a meshed North Sea HVDC grid, Proceedings of the IEEE 101 (4) (2013) 978-990. doi:10.1109/JPROC.2013.2241375.

[6] V. Staudt, A. Steimel, M. Kohlmann, M. K. Jger, C. Heising, D. Meyer, K. Vennemann, E. Grebe, K. Kleinekorte, Control concept including validation strategy for an AC/DC hybrid link (ultranet), in: 2014 IEEE Energy Conversion Congress and Exposition (ECCE), 2014, pp. 750-757. doi:10.1109/ECCE. 2014. 6953471

[7] B. Tourgoutian, A. Alefragkis, Design considerations for the COBRAcable HVDC interconnector, in: IET International Conference on Resilience of Transmission and Distribution Networks (RTDN 2017), 2017, pp. 1-7. doi:10.1049/cp. 2017. 0332.

[8] P. Rault, Implementation of a dedicated control to limit adverse interaction in multi-vendor HVDC systems, IET Conference Proceedings (2019) 7 (6 pp.)-7 (6 pp.)(1).

[9] E. Prieto-Araujo, A. Egea-Alvarez, S. Fekriasl, O. GomisBellmunt, DC voltage droop control design for multiterminal HVDC systems considering AC and DC grid dynamics, IEEE Transactions on Power Delivery 31 (2) (2016) 575-585. doi:10.1109/TPWRD.2015.2451531.

[10] G. O. Kalcon, G. P. Adam, O. Anaya-Lara, S. Lo, K. Uhlen, Small-signal stability analysis of multi-terminal VSC-based DC transmission systems, IEEE Transactions on Power Systems 27 (4) (2012) 1818-1830. doi:10.1109/TPWRS.2012.2190531.

[11] G. Pinares, L. B. Tjernberg, L. A. Tuan, C. Breitholtz, A. A. Edris, On the analysis of the dc dynamics of multi-terminal VSC-HVDC systems using small signal modeling, in: PowerTech (POWERTECH), 2013 IEEE Grenoble, 2013, pp. 1-6. doi:10.1109/PTC.2013.6652303.

[12] S. Cole, J. Beerten, R. Belmans, Generalized dynamic VSC MTDC model for power system stability studies, IEEE Transactions on Power Systems 25 (3) (2010) 1655-1662. doi: 10.1109/TPWRS. 2010.2040846.

[13] J. Beerten, S. D'Arco, J. A. Suul, Identification and small-signal analysis of interaction modes in VSC MTDC systems, IEEE Transactions on Power Delivery 31 (2) (2016) 888-897. doi: 10.1109/TPWRD. 2015.2467965.

[14] M. Amin, A. Rygg, M. Molinas, Impedance-based and eigenvalue based stability assessment compared in VSC-HVDC system, in: 2016 IEEE Energy Conversion Congress and Exposition (ECCE), 2016, pp. 1-8. doi:10.1109/ECCE. 2016.7855185.

[15] T. Qoria, T. Prevost, G. Denis, F. Gruson, F. Colas, X. Guillaud, Power converters classification and characterization in power transmission systems.

[16] A. J. Agbemuko, J. L. Domínguez-García, E. Prieto-Araujo, O. Gomis-Bellmunt, Dynamic modelling and interaction analysis of multi-terminal VSC-HVDC grids through an impedancebased approach, International Journal of Electrical Power \&
Energy Systems 113 (2019) 874 - 887. doi:https://doi.org/ 10.1016/j.ijepes.2019.06.029.

17] M. Amin, M. Molinas, A gray-box method for stability and controller parameter estimation in HVDC-connected wind farms based on nonparametric impedance, IEEE Transactions on Industrial Electronics 66 (3) (2019) 1872-1882. doi:10.1109/TIE. 2018. 2840516.

[18] L. Harnefors, M. Bongiorno, S. Lundberg, Input-admittance calculation and shaping for controlled voltage-source converters, IEEE Transactions on Industrial Electronics 54 (6) (2007) 3323-3334. doi:10.1109/TIE. 2007.904022.

[19] F. D. Freijedo, E. Rodriguez-Diaz, M. S. Golsorkhi, J. C. Vasquez, J. M. Guerrero, A root-locus design methodology derived from the impedance/admittance stability formulation and its application for lcl grid-connected converters in wind turbines, IEEE Transactions on Power Electronics 32 (10) (2017) 82188228. doi:10.1109/TPEL. 2016.2645862.

[20] X. Wang, Y. W. Li, F. Blaabjerg, P. C. Loh, Virtual-impedancebased control for voltage-source and current-source converters, IEEE Transactions on Power Electronics 30 (12) (2015) 70197037. doi : 10.1109/TPEL . 2014. 2382565.

[21] L. Harnefors, A. G. Yepes, A. Vidal, J. Doval-Gandoy, Passivity-based controller design of grid-connected vscs for prevention of electrical resonance instability, IEEE Transactions on Industrial Electronics 62 (2) (2015) 702-710. doi:10.1109/TIE. 2014.2336632.

[22] R. Pea-Alzola, M. Liserre, F. Blaabjerg, M. Ordonez, Y. Yang, Lcl-filter design for robust active damping in grid-connected converters, IEEE Transactions on Industrial Informatics 10 (4) (2014) 2192-2203. doi:10.1109/TII.2014.2361604.

[23] D. Yang, X. Ruan, H. Wu, Impedance shaping of the gridconnected inverter with lcl filter to improve its adaptability to the weak grid condition, IEEE Transactions on Power Electronics 29 (11) (2014) 5795-5805. doi:10.1109/TPEL.2014.2300235.

[24] Z. Xin, P. C. Loh, X. Wang, F. Blaabjerg, Y. Tang, Highly accurate derivatives forlcl-filtered grid converter with capacitor voltage active damping, IEEE Transactions on Power Electronics 31 (5) (2016) 3612-3625. doi:10.1109/TPEL. 2015.2467313.

[25] X. Wang, F. Blaabjerg, M. Liserre, Z. Chen, J. He, Y. Li, An active damper for stabilizing power-electronics-based ac systems, IEEE Transactions on Power Electronics 29 (7) (2014) 33183329. doi:10.1109/TPEL . 2013. 2278716.

[26] M. Cspedes, J. Sun, Impedance shaping of three-phase gridparallel voltage-source converters, in: 2012 Twenty-Seventh Annual IEEE Applied Power Electronics Conference and Exposition (APEC), 2012, pp. 754-760. doi:10.1109/APEC. 2012. 6165904.

[27] J. He, Y. W. Li, Generalized closed-loop control schemes with embedded virtual impedances for voltage source converters with LC or LCL filters, IEEE Transactions on Power Electronics 27 (4) (2012) 1850-1861. doi:10.1109/TPEL. 2011.2168427.

[28] S. Cóbreces, X. Wang, J. Pérez, R. Griñó, F. Blaabjerg, Robust admittance shaping approach to grid current harmonic attenuation and resonance damping, IEEE Transactions on Industry Applications 54 (5) (2018) 5039-5053. doi:10.1109/TIA.2018. 2845358

[29] C. Kammer, S. DArco, A. G. Endegnanew, A. Karimi, Convex optimization-based control design for parallel grid-connected inverters, IEEE Transactions on Power Electronics 34 (7) (2019) 6048-6061. doi:10.1109/TPEL.2018.2881196.

[30] J. Pérez, S. Cobreces, R. Gri, F. J. R. Snchez, $H_{\infty}$ current controller for input admittance shaping of VSC-based grid applications, IEEE Transactions on Power Electronics 32 (4) (2017) 3180-3191. doi:10.1109/TPEL. 2016.2574560.

[31] I. J. Gabe, V. F. Montagner, H. Pinheiro, Design and implementation of a robust current controller for VSI connected to the grid through an LCL filter. IEEE Transactions on Power Electronics 24 (6) (2009) 1444-1452. doi:10.1109/TPEL.2009. 2016097.

[32] S. Cobreces, E. J. Bueno, F. J. Rodriguez, D. Pizarro, F. Huerta, Robust loop-shaping $H_{\infty}$ control of LCL-connected grid con- 
verters, in: 2010 IEEE International Symposium on Industrial Electronics, 2010, pp. 3011-3017. doi:10.1109/ISIE.2010. 5637614.

[33] A. J. Agbemuko, J. L. Domínguez-García, O. Gomis-Bellmunt, An integrated approach to understanding the impact of network resonances and control on dynamic responses in VSC-HVdc networks, IFAC-PapersOnLine 51 (28) (2018) 344 - 349, 10th IFAC Symposium on Control of Power and Energy Systems CPES 2018. doi:https://doi.org/10.1016/j.ifacol.2018.11.726.

[34] A. Yazdani, R. Iravani, Voltage-sourced converters in power systems: modeling, control, and applications, John Wiley \& Sons, 2010.

[35] J. Beerten, Frequency-dependent cable modelling for smallsignal stability analysis of vsc-hvdc systems, IET Generation, Transmission \& Distribution 10 (2016) 1370-1381(11).

[36] A. J. Agbemuko, J. L. Domínguez-García, E. Prieto-Araujo, O. Gomis-Bellmunt, Advanced impedance-based control design for decoupling multi-vendor converter HVDC grids, (Under Review) IEEE Transactions on Power Delivery.

[37] E. Bristol, On a new measure of interaction for multivariable process control, IEEE Transactions on Automatic Control 11 (1) (1966) 133-134. doi:10.1109/TAC.1966.1098266.

[38] S. Skogestad, I. Postlethwaite, Multivariable feedback control: analysis and design, Vol. 2, Wiley New York, 2007.

[39] P. Gahinet, P. Apkarian, Decentralized and fixed-structure $\mathrm{H}_{\infty}$ control in matlab, in: 2011 50th IEEE Conference on Decision and Control and European Control Conference, 2011, pp. 82058210. doi:10.1109/CDC.2011.6160298. 